$\Rightarrow$ LIVER

\title{
Clearance of nanomaterials in the liver
}

A mechanism for how hard nanomaterials are transported and cleared by the liver has been proposed in new research published in Nature Materials. The findings show important roles for flow dynamics, organ microarchitecture and cellular phenotypes in this process.

"Nanoparticles are designed to diagnose and treat diseases but the main problem is that when they are introduced in the bloodstream, over $99 \%$ of them are cleared by cells in the liver and other organs of the reticuloendothelial system," explain authors Warren Chan and Ian McGilvray. "This presents a problem for using nanoparticles for medical applications, as very few of them will be able to interact with the diseased tissue." Chan, McGilvray and colleagues used a multidisciplinary approach to determine how the liver mediates this clearance process.

In a series of tests (including in vivo experiments and mathematical modelling), the researchers examined the clearance of hard, nondegradeable nanomaterials (quantum dots, gold nanoparticles and silica nanoparticles). They found that when nanomaterials enter and traverse the liver, their velocity reduces 1,000 -fold, increasing the probability of interactions between nanomaterials and liver cells, and then subsequent clearance. Surprisingly, once inside the liver, nanomaterials are taken up and cleared by a variety of cells - primarily Kupffer cells, hepatic B cells and liver sinusoidal endothelial cells — and not just Kupffer cells as expected.

The investigators are planning further research into how resident liver cells and nanoparticles interact, as well as potential strategies to overcome liver sequestration of nanomaterials.

Katrina Ray

ORIGINAL ARTICLE Tsoi, K. M. et al. Mechanism

of hard-nanomaterial clearance by the liver. Nat. Mater. http://dx.doi.org/10.1038/nmat4718 (2016) 